\title{
Association between antidepressant resistance in unipolar depression and subsequent bipolar disorder: cohort study ${ }^{\dagger}$
}

\author{
Cheng-Ta Li, Ya-Mei Bai, Yu-Lin Huang, Ying-Sheue Chen, Tzeng-Ji Chen, Ju-Yin Cheng \\ and Tung-Ping Su
}

\section{Background}

People with major depressive disorder who fail to respond to adequate trials of antidepressant treatment may harbour hidden bipolar disorder.

\section{Aims}

We aimed to compare the rates of a change in diagnosis to bipolar disorder among people with major depressive disorder with stratified responses to antidepressants during an 8-year follow-up period.

\section{Method}

Information on individuals with major depressive disorder identified during 2000 (cohort 2000, $n=1485$ ) and 2003 (cohort 2003, $n=2459$ ) were collected from a nationally representative cohort of 1000000 health service users in Taiwan. Participants responding well to antidepressants were compared with those showing poor responses to adequate trials of antidepressants.

\section{Results}

In 7.6-12.1\% of those with a diagnosis of unipolar major depressive disorder this diagnosis was subsequently changed to bipolar disorder, with a mean time to change of 1.89-2.98 years. Difficult-to-treat participants presented higher rates of change to a bipolar diagnosis $(25.6 \%$ in cohort 2000; $26.6 \%$ in cohort 2003) than easy-to-treat participants $(8.8-8.9 \%$ in cohort 2000; 6.8-8.6\% in cohort 2003; $P<0.0001)$. Regression analysis showed that the variable most strongly associated with the change in diagnosis was antidepressant use history. The difficult-to-treat participants were associated most with diagnostic changing (cohort 2000: odds ratio $(\mathrm{OR})=1.88(95 \% \mathrm{Cl}$ 1.12-3.16); cohort 2003: $\mathrm{OR}=4.94$ (95\% Cl 2.81-8.68)).

\section{Conclusions}

This is the first large-scale study to report an association between antidepressant response history and subsequent change in diagnosis from major depressive disorder to bipolar disorder. Our findings support the view that a history of poor response to antidepressants in unipolar depression could be a useful predictor for bipolar diathesis.

\section{Declaration of interest}

None.
Unipolar major depressive disorder is a leading cause of disease burden around the world because of its chronic and recurrent nature. ${ }^{1}$ Up to $50 \%$ of people with depression may remain depressed, or have at least one recurrence during follow-up periods. ${ }^{2}$ However, a substantial proportion of people with depression fail to respond to adequate trials of antidepressants. ${ }^{3,4}$ Only a few individuals seem to achieve complete symptomatic remission. ${ }^{1,3}$ In addition, partial or even no response to antidepressants in people with major depressive disorder is estimated to be over $30 \%{ }^{4}$ Medication-resistant depression is not only common, but also leads to worsened outcomes, including more hospitalisations and even enhanced mortality rates. ${ }^{1,4,5}$

Unrecognised or hidden bipolar disorder could be related to medication resistance in unipolar depression. ${ }^{6-9}$ The course of bipolar disorder usually begins with depression and could be diagnosed as major depressive disorder at the initial stage. This kind of hidden bipolar disorder may contribute to the treatment resistance observed in unipolar depression. ${ }^{9}$ Likewise, a poor response to several adequate antidepressant treatment trials in unipolar depression has also been suggested to be a soft sign of bipolar disorder. ${ }^{10}$ Previous results showed that more than 50\% of the people with unipolar treatment-resistant depression were subsequently diagnosed with occult bipolar disorder when reappraised during the follow-up period., ${ }^{9,11}$ However, the lack of masking in the prospective studies may have resulted in investigator bias and the misinterpretation of symptoms as hypomania or mania, and almost all the published research

'See editorial, pp. 5-6, this issue studies utilised a relatively small sample size. Therefore, large-scale studies directly delineating the association between antidepressant resistance in unipolar depression and hidden bipolar disorder are still lacking.

The present study aimed to compare changes in the rate at which bipolar disorder was diagnosed during an 8-year follow-up period (2000-2007) among people with major depressive disorder with different historical patterns of antidepressant response. We utilised the Taiwanese nationwide database of National Health Insurance (NHI) claims. Our primary hypothesis was that people with major depressive disorder who showed more antidepressant resistance would have a higher rate of change in diagnosis to that of bipolar disorder, compared with those showing seemingly better responses to antidepressants. Clinicians in Taiwan have gradually increased their recognition of bipolar disorder, as reflected by the slightly increased prevalence rates reported for bipolar disorder (year 2000: 0.16\%; and year 2003: 0.21\%). ${ }^{12}$ We therefore sought also to investigate whether clinicians' awareness might have influenced the changes in the bipolar diagnostic rates.

\section{Method}

\section{Data sources}

We utilised the NHI database covering a period from 1996 to 2007, which was published by the National Health Research Institutes of Taiwan. Taiwan has run this NHI programme since 1995 to finance healthcare for all its residents. The coverage rate was $96.16 \%$ of the whole population in 2000 and this had risen 
to $99 \%$ by the end of 2004 . The completeness and accuracy of the NHI database are guaranteed by the Department of Health and the Bureau of NHI of Taiwan. ${ }^{13}$ The database comprises comprehensive information about clinical visits for each insured person, such as demographic data, date of visits, diagnostic codes according to the clinical modification of the ICD-9 (ICD-9-CM) ${ }^{14}$ and prescription details. Since the data were released for research purposes only, and because the identities of the participants and physicians were scrambled, the study was exempt from a full review by the local ethics review committee.

\section{Participants}

The selection criteria for participants are shown in Fig. 1. Our cohort 2000 study included all individuals in Taiwan who were diagnosed with major depressive disorders (ICD-9-CM code: 296.2 and 296.3) between January and December 2000. To prevent misdiagnosis of major depression, we excluded individuals diagnosed with bipolar disorder (ICD-9-CM code: 296.0, 296.1, 296.4, 296.5, 296.6, 296.7 and 296.8) between 1996 and 1999. Affective psychosis (ICD-9-CM code: 296.9) was also excluded because it reduces the distinctions between unipolar and bipolar disorders to such an extent that an accurate diagnosis becomes too unreliable. To further ensure the validity of the diagnosis for major depressive disorder, we excluded individuals who were not diagnosed by a psychiatrist, and only included those individuals who were diagnosed with major depressive disorder by a psychiatrist on at least two occasions between January and December 2000. To try to duplicate our findings, we identified another cohort (cohort 2003) and applied the same method outlined above, except over the period January to December 2003.

\section{Stratification according to patterns of antidepressant use history}

In order to test our primary hypothesis, all study participants were further stratified according to their antidepressant use characteristics during a period extended from 1 year before to 1 year after the study year, so for cohort 2000, over 1999-2001 and for cohort 2003, over 2002-2004. Depression is usually considered resistant to antidepressants when at least two trials of different antidepressants (adequate in terms of dosage and duration) fail to produce a significant clinical improvement. In the present study, we defined participants as difficult to treat (DTT) if their antidepressant treatment regime was altered two or more times. An adequate trial is defined as using an antidepressant within its therapeutic dosage range (for example, fluoxetine $\geqslant 20 \mathrm{mg} /$ day) for more than 60 consecutive days. Our comparison groups, which contained participants who were considered relatively easy to treat (ETT), included one group of individuals not taking any antidepressants (ETT-1) and a second group who stayed on a single antidepressant (ETT-2). Patients who changed their antidepressants, but only once, were defined as intermediate difficult to treat (ITT). To summarise, we categorised the participants into four groups according to their response to antidepressants, namely ETT-1, ETT-2, ITT and DTT.

\section{Identification of patients whose diagnosis changed to bipolar disorder}

The selection criteria for bipolar disorder are shown in Fig. 1. To survey participants for a change in diagnosis from major depressive disorder to bipolar disorder over time, we screened for individuals with major depressive disorder between 2000 and 2007 in the cohort 2000 study, and between 2003 and 2007 in the cohort 2003 study. To ensure the validity of the diagnosis of bipolar disorders (ICD-9-CM code: 296.0, 296.1, 296.4, 296.5, 296.6, 296.7 and 296.8), we excluded people whose diagnosis of bipolar disorder was not made by psychiatrists, and only included those who were diagnosed with bipolar disorder by psychiatrists on at least two occasions. The outcome measurements were (a) mean (s.d.) time to a change in diagnosis from major depressive disorder to bipolar disorder; and (b) the rate and percentage of

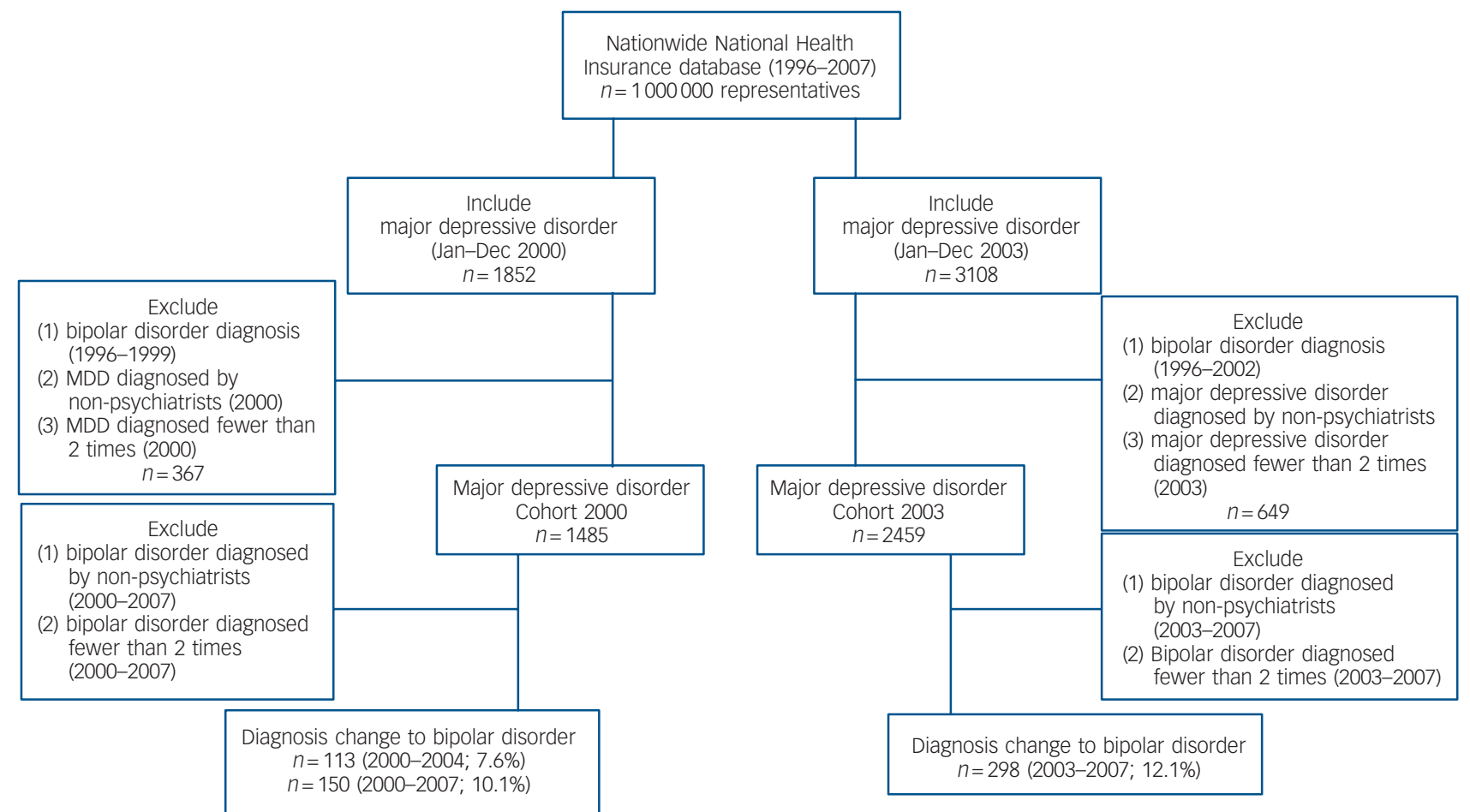

Fig. 1 Flow chart of sample selection criteria. MDD, major depressive disorder. 
such changes of diagnosis over time among the four groups with increasing levels of treatment difficulties.

\section{Prevalence of bipolar disorder from 2000 to 2007}

We compared the prevalence of bipolar disorder from 2000 to 2007. In this comparison, we identified every bipolar disorder diagnosis reported by all the clinicians, since our objective was to investigate changes in bipolar disorder prevalence over time.

To explore the bipolar disorder subtypes, participants with more than two psychiatric hospitalisations following a change in diagnosis from major depressive disorder to bipolar disorder were assumed to have bipolar I disorder, otherwise a diagnosis of bipolar II disorder was assigned. The assumption was that people with bipolar I disorder would have had more psychiatric hospitalisations for manic episodes than those with subtype II, since an objective criterion to differentiate mania from hypomania is the need to receive in-patient treatment for manic symptoms.

\section{Other independent variables}

Dysthymia, anxiety (anxiety states and anxiety disorders) and substance misuse or dependence are highly comorbid with major depression. These comorbidities were also studied to avoid a confounding effect on antidepressant history used to predict the subsequent change of diagnosis to bipolar disorder. Dysthymia (ICD-9-CM code: 300.4), anxiety states $(300.0,300.00,300.09$, $300.1,300.15,300.8,300.89$ and 300.9), anxiety disorders, including panic disorder (300.01), agoraphobia with panic attacks (300.21), generalised anxiety disorder (300.02), social phobia (300.23), obsessive-compulsive disorders (300.3), neurasthenia (300.5), hypochondriasis (300.7) and somatisation disorder (300.81), were identified. Regarding substance misuse and dependence, alcohol misuse and dependence (303, 303.9, $303.9 \mathrm{X}, 305.0 \mathrm{X})$ as well as other substance misuse and dependences such as opioid (304.0X, 305.5X), barbiturate (304.1X, 305.4X), cocaine (304.2X, 305.6X), cannabis (304.3X, 305.2X), amphetamine (304.4X, 305.7X) and hallucinogen $(304.5 \mathrm{X}, 305.3 \mathrm{X})$ were also identified in both cohort 2000 and cohort 2003 studies.

Other variables, including age, gender and antidepressant monotherapy or combination therapy, were analysed. We aimed to see whether the different groups (by response to antidepressants: ETT-1, ETT-2, ITT and DTT) and these variables during the index period (for example 1999-2001 in the cohort 2000 study) would have a predictive value for the subsequent change of diagnosis to bipolar disorder (for example, 2000-2007 in the cohort 2000 study).

\section{Statistical significance}

The SAS statistical package (SAS System for Windows, version 9.2) and SPSS statistics (SPSS for Windows, version 17.0) were used to perform the statistical analysis of the data in the present study. Categorical variables (for example, the change in diagnosis, gender, anxiety and substance comorbidity) between groups were analysed by chi-squared tests. Continuous variables such as age were analysed by independent $t$-tests or one-way ANOVA. To prevent collinearity between independent variables, correlation analysis between categorical variables was done by chi-squared tests. Thus, multivariate logistic regression was carried out, with age, education and the groups with different levels of treatment difficulty treated as independent factors and change of diagnosis from major depressive disorder to bipolar disorder as the dependent factor. Adjusted odds ratios and 95\% confidence intervals were reported for the risk of change of diagnosis, and $P<0.05$ (2-sided tests) was deemed to be statistically significant.

\section{Results}

As described above, we divided participants into four groups according to their antidepressant use history. Age and gender among these four groups in cohort 2000 and cohort 2003 did not differ statistically (Table 1). As shown in Table 1, $80 \%$ (cohort 2000) and 70.9\% (cohort 2003) of participants were either not treated with any antidepressants (ETT-1) or were only treated with one type of antidepressant (ETT-2). However, around 5.5\% (cohort 2000) and $10.7 \%$ (cohort 2003) of participants showed higher resistance to antidepressants, since they failed to respond to adequate antidepressant trials two or more times during the index period (Table 1).

We found that $7.6 \%$ (within 5 years in cohort 2000), 10.1\% (within 8 years in cohort 2000) and $12.1 \%$ (within 5 years in cohort 2003) of the participants originally diagnosed with unipolar depression eventually had their diagnosis changed to bipolar disorder (Fig. 1). Participants who showed antidepressant resistance (DTT), i.e. a past history of changing antidepressant after an adequate antidepressant trial on at least two occasions, presented the highest rate of change of diagnosis (25.6\% in cohort 2000 and $26.6 \%$ in cohort 2003) compared with the three other groups $(P<0.0001)$, as shown in Table 2. Participants who had an antidepressant history indicative of no use at all (ETT-1) or of a single antidepressant (ETT-2), presented significantly lower rates of change of diagnosis (for example, $8.9 \%$ for ETT-1 and $8.8 \%$ for ETT-2 in the cohort 2000). The ITT group, which included participants with a single change of

\begin{tabular}{|c|c|c|c|c|}
\hline Groups & Participants, n (\%) & Age, years: mean (s.d.) & Males, $n(\%)$ & Females, $n(\%)$ \\
\hline \multicolumn{5}{|c|}{ Cohort $2000(n=1485)$} \\
\hline ETT-1 & $237(16.0)$ & $47.5(17.1)$ & 76 & 161 \\
\hline ETT-2 & $950(64.0)$ & $47.6(16.6)$ & 344 & 606 \\
\hline $\mathrm{ITT}$ & 189 (12.7) & $48.1(14.9)$ & 72 & 117 \\
\hline $\mathrm{DTT}$ & $82(5.5)$ & $46.4(15.6)$ & 35 & 47 \\
\hline Total & $1458(98.2)$ & - & $527(35.5)$ & $931(62.7)$ \\
\hline \multicolumn{5}{|c|}{ Cohort $2003(n=2459)$} \\
\hline ETT-1 & $162(6.6)$ & $46.8(18.2)$ & 65 & 97 \\
\hline ETT-2 & $1580(64.3)$ & $46.8(17.8)$ & 544 & 1036 \\
\hline $\mathrm{ITT}$ & $395(16.1)$ & $46.7(16.8)$ & 141 & 254 \\
\hline $\mathrm{DTT}$ & $263(10.7)$ & $44.7(14.5)$ & 99 & 164 \\
\hline Total & $2400(97.6)$ & - & 849 (34.5) & $1551(63.1)$ \\
\hline
\end{tabular}


antidepressant after an adequate antidepressant trial, showed an intermediate rate of subsequent change of diagnosis. The rates of change of diagnosis among the four groups were statistically significant $(P<0.0001)$ (Table 2$)$. The rates of change over time are illustrated in Fig. 2. The cumulative rates from 2000 to 2007 were faster and higher rates in the DTT group, whether in cohort 2000 or cohort 2003. Other variables that significantly influenced change of diagnosis included younger age and antidepressant combinations, but not gender or psychiatric comorbidity (Table 2).

No statistical significance relating to psychiatric comorbidity existed between these two cohorts, but more than $50 \%$ of the participants had comorbid dysthymia, anxiety and substance use problems (Table 3 ). In cohort 2000, 33.4\% of participants had comorbid dysthymia, $63.3 \%$ had anxiety $(50.1 \%$ : anxiety states and $13.2 \%$ : anxiety disorders) and $3.3 \%$ substance misuse/ dependence. Likewise in cohort 2003,30.9\% of participants were noted as having dysthymia, $64.5 \%$ anxiety and $4.6 \%$ substance use problems.

There were significant interactions between groups (ETT-1, ETT-2, ITT and DTT) and antidepressant combinations as well as psychiatric comorbidity (online Table DS1). Therefore, the multivariate logistic regression model only included the following variables: age, gender and groups with different levels of treatment difficulty. The adjusted odds ratios for both cohort 2000 and cohort 2003 for change of diagnosis are shown in Table 2. Age was associated with change of diagnosis (Wald $\chi^{2}=4.14$, d.f. $=1$, $P=0.042$ ), but a weak odds ratio (0.99) was found. The variable most strongly associated with diagnostic change was antidepressant use history: in cohort 2000, for the DTT group Wald $\chi^{2}=4.40$, d.f. $=1, P=0.036$, OR $=1.88$ (95\% CI 1.12-3.16). The association was even stronger in the 2003 cohort: Wald $\chi^{2}=14.8$, d.f. $=1, \quad P<0.0001, \quad O R=4.94 \quad$ (95\% CI 2.81-8.68). Similar results were found in the associations between the ITT group and risk of diagnostic change, both in cohort 2000 and cohort 2003. These findings support the theory that antidepressant resistance history in unipolar depression is a useful predictor of vulnerability to developing bipolar disorder later in life.

The mean time to change of diagnosis was 2.98 years $($ s.d. $=2.34)$ within 8 years $($ cohort 2000); 2.07 years $($ s.d. $=1.52)$ within 5 years (cohort 2000); and 1.89 years $($ s.d. $=1.31)$ within 5 years (cohort 2003). The rate of diagnostic changing was also faster in the 2003 cohort. For example, for the DTT groups, $3.5 \%$ of diagnoses were changed per year in cohort 2000 and $5.3 \%$ per year in cohort 2003. Since clinicians' awareness of bipolar disorder would have influenced the rate of diagnostic changing, we also investigated the prevalence of bipolar disorder from 2000 to 2007 . We found that the prevalence of bipolar disorder in Taiwan increased from $0.186 \%$ in 2000 to $0.283 \%$ in 2007 (online Fig. DS1). The increase was most drastic between 2003 and 2005. This explains not only the faster rate of change of diagnosis in the 2003 cohort, but also the enhanced slope of the cumulative diagnostic changing rates between 2004 and 2006 in cohort 2000 (Fig. 2(a)). These results support the notion that increased clinical awareness of bipolar disorder over time was an important factor in raising the rates of change of diagnosis even further.

Finally, we explored the subtypes of bipolar disorder. We found that $12.4 \%$ (within 5 years; 14/113) and $14.0 \%$ (within 8 years; 21/150) of participants in cohort 2000 had bipolar I disorder and only 6.7\% (within 5 years; 20/298) in cohort 2003 had bipolar I disorder. This indicated that the enhanced number of individuals whose diagnosis was changed to bipolar disorder found in the cohort 2003 study mostly had bipolar II disorder.

\section{Discussion}

\section{Main findings}

This is the first large-scale study investigating the association between antidepressant use history in unipolar major depressive disorder and hidden bipolar disorder using a nationwide database. First, our findings support our hypothesis that people with major depressive disorder who show more antidepressant resistance have higher subsequent rates of a change in diagnosis to bipolar disorder. The supporting evidence is that individuals who are relatively difficult to treat (our DTT and ITT groups) had a higher risk of subsequent change of diagnosis than those who are easy to treat (ETT-1 and ETT-2). It has been a widespread clinical belief that unrecognised bipolar disorder is a frequent contributor to treatment-resistant depression ${ }^{15}$ and our results directly support this idea. Second, we found that $7.6-12.1 \%$ of participants with major depressive disorder had their diagnosis changed, after a mean time of 1.89-2.98 years. Finally, we found that clinicians' awareness of bipolar disorder would have increased the rate and shortened the time to change of diagnosis.

The strengths of the current study are the large sample size drawn from a nationwide database and hence without investigator bias in judging bipolar symptoms. By using this database, covering a representative cohort of 1000000 health service users (19962007), we corroborated that unipolar major depressive disorder
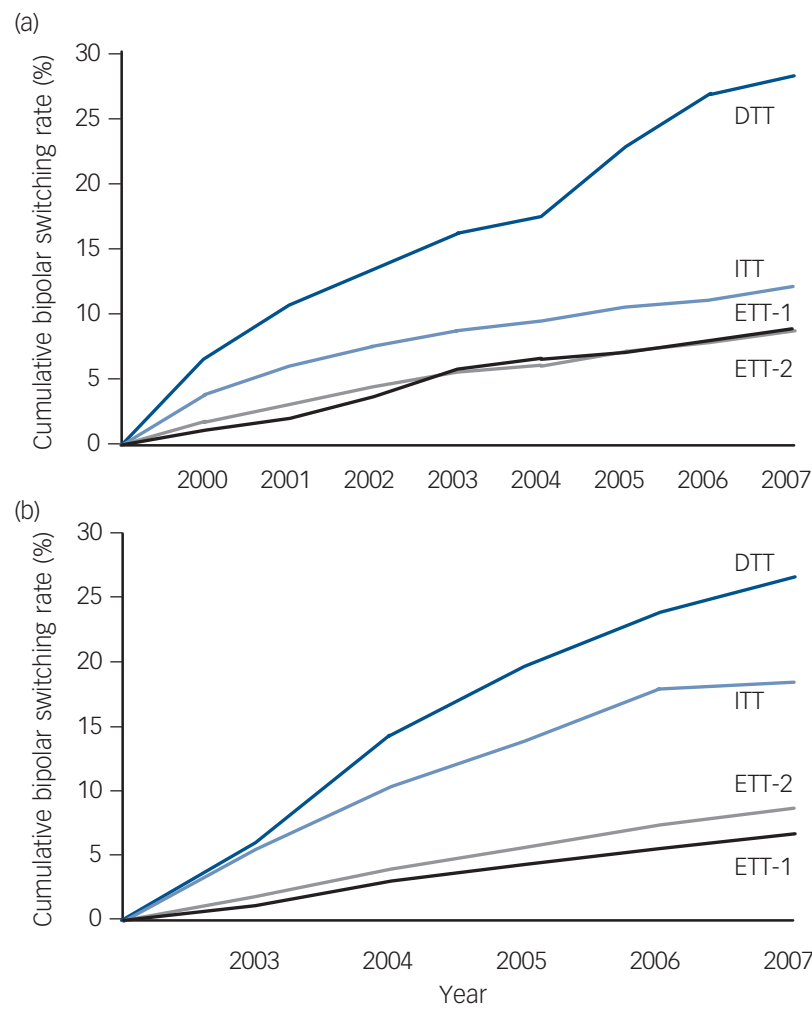

Fig. 2 Rates of change in diagnosis from major depressive disorder to bipolar disorder over time. There are four groups with different levels of antidepressant response history in both (a) cohort 2000 and (b) cohort 2003.

Participants with medication-resistant history (difficult-to-treat group (DTT)) exhibit the highest rates of diagnostic change during the subsequent 8 years, as compared with those without any antidepressant use (easy-to-treat group 1 (ETT-1)) or those without any change in antidepressant (easy-to-treat group 2 (ETT-2)). Participants who changed antidepressant just once, after an adequate antidepressant trial (intermediate level of difficulty to treat (ITT)) display intermediate rates of change. Both cohorts show similar patterns in these four groups, with DTT showing the highest rate of diagnostic change throughout the follow-up. Participants with ETT-1 and ETT-2 did not show different rates of change. 


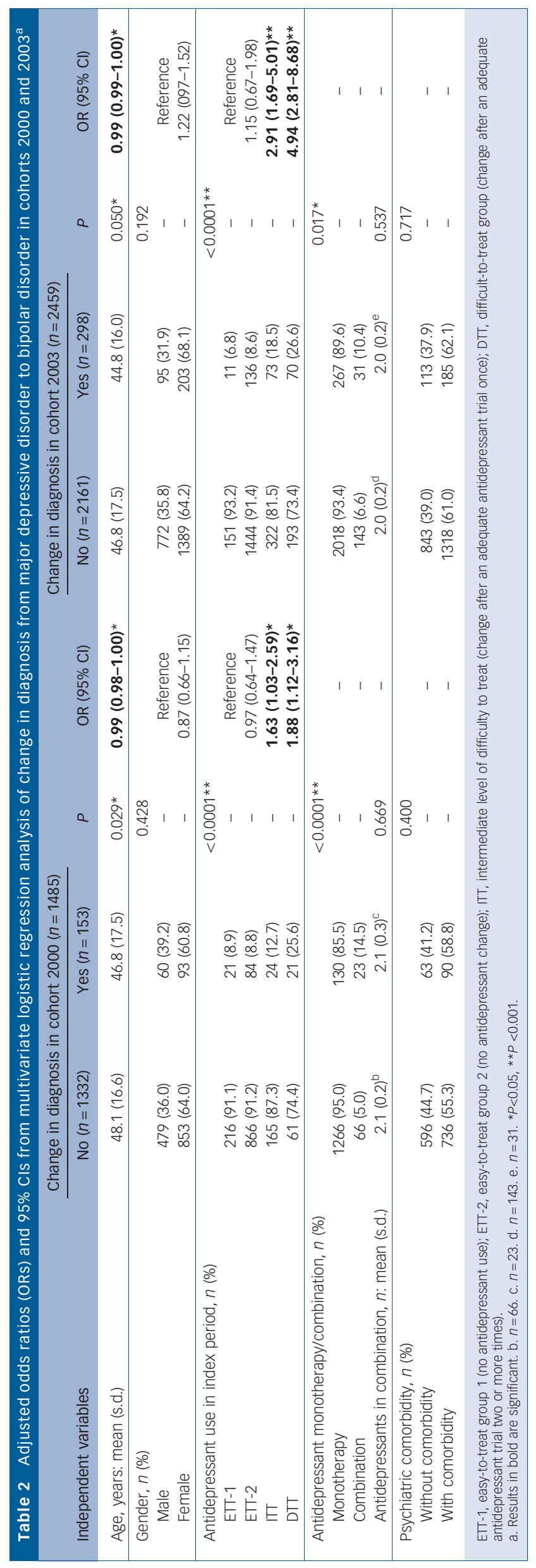

with a poor response to adequate antidepressant trials could be a sign of hidden bipolar disorder. ${ }^{9}$ The rate could be as high as $50 \%$ in treatment-resistant depression, based on the results from prospective observational studies. ${ }^{9,11,16}$ However, we found a lower rate for the change in diagnosis in our DTT group (25.6$26.6 \%)$. One explanation for this discrepancy is the lack of masking in the prospective naturalistic studies and therefore the risk of bias in the investigator's judgement in discovering bipolarity in individuals with treatment-resistant depression. Another explanation is that bipolar disorder may have been underdiagnosed because some individuals had hypomanic or manic symptoms that were not severe or were not clinically relevant.

The major finding of the present study was that participants with easy-to-treat unipolar major depressive disorder (ETT-1 and ETT-2 groups) were less likely to convert to bipolar disorder over time, whereas those who were difficult to treat (DTT and ITT groups) were more likely to have a change of diagnosis. The rates of diagnostic change in our four groups of participants were ordered in a dose-dependent pattern. The easy-to-treat ones (ETT-1 and ETT-2) had lower rates of change (6.8-8.8\%) than the difficult-to-treat ones (DTT, 25.6-26.6\%). Participants who switched antidepressants only once (ITT) fell in between these limits (12.7-18.5\%). Our findings support the view that medication-resistant depression is the link between unipolar and bipolar disorders, especially bipolar II disorder. Depression with poor or partial responses to antidepressants is common ${ }^{17-20}$ and is one of the major challenges for both patients and clinicians. Early detection and adequate intervention are our goals in treating individuals. To date, a growing body of evidence supports a link between medication- resistant major depressive disorder and bipolar disorder. For example, in a study using bipolar screening questionnaires it was found that individuals with treatmentresistant depression scored significantly higher than those whose depression was not treatment resistant. ${ }^{21}$ Regarding the medication options for antidepressant-resistant depression, augmentation of psychotropic medications with mood stabilisers (for example, lithium) and atypical antipsychotics (for example, aripiprazole, olanzapine, quetiapine and risperidone) is more effective than antidepressant monotherapy. ${ }^{18-20}$ The effectiveness of using medications for bipolar disorder to treat medication-resistant depression also provides indirect support for the notion that medication-resistant unipolar depression could be the link between unipolar and bipolar disorders.

The second major finding of the present study was that a reproducible approximately $10 \%$ of all individuals with unipolar depression are likely to switch to bipolar disorder over time. We found that for $7.6-12.1 \%$ of participants diagnosed with unipolar major depressive disorder the diagnosis was changed to bipolar disorder over time. This is consistent with some previous prospective studies. ${ }^{22-24}$ For example, Akiskal et al conducted an 11 -year prospective study, finding that $12.5 \%$ (70/559) of people with unipolar depression were subsequently diagnosed with bipolar disorder. ${ }^{22}$ Another research study conducted in Finland yielded similar results, in which they found that $11.7 \%$ (29/248) of individuals previously diagnosed with unipolar major depressive disorder were later diagnosed with bipolar disorder during a 5 -year period. ${ }^{24}$ Although a longer follow-up period has been suggested to contribute to more diagnostic switching, our data support that the level of treatment difficulty could be a more reliable variable in predicting bipolar disorder diathesis. For example, one previous prospective research study conducted in psychiatric in-patients found a slightly higher diagnostic changing rate of $15.5 \%{ }^{25}$ This higher rate could be explained by the special focus placed on individuals hospitalised in Switzerland with severe major depressive disorder. 


\begin{tabular}{|c|c|c|c|}
\hline Psychiatric comorbidity & $\begin{array}{l}\text { Comorbid in cohort } 2000 \\
\qquad(n=1485)\end{array}$ & $\begin{array}{l}\text { Comorbid in cohort } 2003 \\
\qquad(n=2459)\end{array}$ & $P$ \\
\hline Dysthymia, $n$ (\%) & $276(33.4)$ & $398(30.9)$ & NS \\
\hline Anxiety, $n(\%)$ & & & NS \\
\hline Anxiety states & $414(50.1)$ & $577(44.8)$ & \\
\hline Anxiety disorders & $109(13.2)$ & $254(19.7)$ & \\
\hline Panic disorder & $33(4.0)$ & $86(6.7)$ & \\
\hline Agoraphobia & $23(2.8)$ & $64(5.0)$ & \\
\hline Generalised anxiety disorder & $7(0.8)$ & $10(0.8)$ & \\
\hline Social phobia & $5(0.6)$ & $10(0.8)$ & \\
\hline Obsessive-compulsive disorder & $30(3.6)$ & $59(4.6)$ & \\
\hline Neurasthenia & $6(0.7)$ & $13(1.0)$ & \\
\hline Hypochondriasis & $2(0.2)$ & $6(0.5)$ & \\
\hline Somatisation disorder & $3(0.4)$ & $6(0.5)$ & \\
\hline Substance misuse and dependence, $n(\%)$ & $27(3.3)$ & $58(4.6)$ & NS \\
\hline Alcohol misuse and dependence & $17(2.1)$ & $29(2.3)$ & \\
\hline Other substance misuse and dependence & $10(1.2)$ & $29(2.3)$ & \\
\hline Total, $n(\%)$ & $826(100)$ & $1287(100)$ & \\
\hline$\%$, relative to whole cohort & 55.6 & 52.3 & \\
\hline
\end{tabular}

It has long been debated whether certain antidepressants, such as tricylic antidepressants (TCAs) and serotonin-noradrenaline reuptake inhibitors (SNRIs), could induce switching to mania. One of the conclusions drawn from the present study is that there is no direct link between the time of the antidepressant screening (for example, 1999-2001 in cohort 2000) and the subsequent diagnostic change (for example, 2 years later). Therefore, we believe that even for people with difficult-to-treat depression prescribed more TCAs or SNRIs than easy-to-treat individuals, a change in diagnosis from major depressive disorder to bipolar disorder could not be explained by the use of such antidepressants because potential manic or hypomanic symptoms only emerged much later after their use. To explore whether TCAs and SNRIs contribute to more changes of diagnosis, we investigated levels of antidepressant use just prior to the diagnostic change to bipolar disorder. We found that the prevalence of TCA or SNRI prescriptions was $30.5 \%, 28.6 \%$ and $36.0 \%$ in the easy-to-treat, the intermediate difficult-to-treat and the difficult-to-treat groups, respectively. The findings suggest that the use of TCAs or SNRIs cannot fully explain the observed phenomenon of a higher diagnostic change rate in the participants with antidepressantresistant depression. The relatively higher prevalence of TCA and SNRI usage in the difficult-to-treat group might be partly related to these individuals' refractoriness to antidepressants.

\section{Limitations}

There are some limitations in this study using a registry-based database. First, a lack of structured interview to diagnose major depressive disorder or bipolar disorder might increase the diagnostic uncertainty. However, we increased our inclusion threshold and identified only participants from the nationwide database who had been diagnosed at least twice by psychiatrists. Second, we cannot truly differentiate between subtypes of bipolar disorders since there is a lack of specific ICD-9-CM codes for the bipolar subtypes. However, we assumed that participants with bipolar I disorder would have more psychiatric hospitalisations because of manic episodes than those with bipolar II disorder. Besides, this is not the primary objective of the present study. Further studies are warranted to resolve this issue. Third, we could not assess drug adherence to truly assess whether the antidepressant trials were adequate. Finally, the percentage of antidepressant-related mood switching cannot be identified. However, antidepressant-induced mania/hypomania is not a universal phenomenon when treating people with depression and has long been referred to as part of the bipolar spectrum. ${ }^{9}$ In addition, we found that $6.8-8.9 \%$ of people with depression without antidepressant usage (group ETT-1) eventually had a diagnostic change to bipolar disorder, and the changing rate was almost the same as for those participants who stayed on a single antidepressant (ETT-2) (8.6-8.8\%).

\section{Implications}

A strong association between poor antidepressant responses and a subsequent change in diagnosis to one of bipolar disorder was found in this study using a nationwide database in Taiwan. Clinicians' awareness of bipolar disorder also increased the rate at which diagnosis was changed and shortened the time to change. Our findings suggest that in individuals with major depression, a history of poor response to antidepressants is a useful sign for predicting bipolar diathesis.

Cheng-Ta Li, MD, Department of Psychiatry, Taipei Veterans General Hospital, Taipe Institute of Brain Science and the Division of Psychiatry, Faculty of Medicine, Nationa Yang-Ming University, Taipei; Ya-Mei Bai, MD, PhD, Department of Psychiatry, Taipei Veterans General Hospital, Taipei and Division of Psychiatry, Faculty of Medicine, National Yang-Ming University, Taipei; Yu-Lin Huang, MD, Department of Dermatology, Faculty of Medicine, National Yang-Ming University, Taipei; Ying-Sheue Chen, MD, Department of Psychiatry, Taipei Veterans General Hospital, Taipei; Tzeng-Ji Chen, MD, PhD, Department of Family Medicine, Taipei Veterans General Hospital, Taipei; Ju-Yin Cheng, MS, Department of Psychiatry, Taipei Veterans General Hospital, Taipei; Tung-Ping Su, MD, Department of Psychiatry, Taipei Veterans General Hospital, Taipei, Institute of Brain Science and the Division of Psychiatry, Faculty of Medicine, National Yang-Ming University, Taipei, Taiwan

Correspondence: Tung-Ping Su, Department of Psychiatry, Taipei Veterans General Hospital, No. 201, Sec. 2, Shih-Pai Road, Taipei, 11217, Taiwan. Email: tomsu0402@gmail.com

First received 14 Nov 2010, final revision 19 Feb 2011, accepted 16 Jun 2011

\section{Funding}

This work is supported by grants from the Taipei Veterans General Hospital (V100D-002-1). 


\section{Acknowledgements}

This study is based in part on data from the National Health Insurance Research Databas provided by the Bureau of National Health Insurance, Department of Health and managed by National Health Research Institutes. The interpretation and conclusions contained here do not represent those of the Bureau of National Health Insurance, Department of Health or National Health Research Institutes.

\section{References}

1 Fava M. Diagnosis and definition of treatment-resistant depression. Biol Psychiatry 2003; 53: 649-59.

2 Eaton WW, Shao $\mathrm{H}$, Nestadt G, Lee HB, Bienvenu OJ, Zandi P. Populationbased study of first onset and chronicity in major depressive disorder. Arch Gen Psychiatry 2008; 65: 513-20.

3 Moller HJ. Outcomes in major depressive disorder: the evolving concept of remission and its implications for treatment. World J Biol Psychiatry 2008; 9 102-14

4 Bschor T. Therapy-resistant depression. Expert Rev Neurother 10: 77-86.

5 Crown WH, Finkelstein S, Berndt ER, Ling D, Poret AW, Rush AJ, et al. The impact of treatment-resistant depression on health care utilization and costs. J Clin Psychiatry 2002; 63: 963-71.

6 Kornstein SG, Schneider RK. Clinical features of treatment-resistant depression. J Clin Psychiatry 2001; 62 (suppl 16): 18-25.

7 Inoue T, Nakagawa S, Kitaichi Y, Izumi T, Tanaka T, Masui T, et al. Long-term outcome of antidepressant-refractory depression: the relevance of unrecognized bipolarity. J Affect Disord 2006; 95: 61-7.

8 Akiskal HS, Mallya G. Criteria for the "soft" bipolar spectrum: treatment implications. Psychopharmacol Bull 1987; 23: 68-73.

9 Correa R, Akiskal H, Gilmer W, Nierenberg AA, Trivedi M, Zisook S. Is unrecognized bipolar disorder a frequent contributor to apparent treatment resistant depression? J Affect Disord 2010; 127: 10-8.

10 Ghaemi SN, Ko JY, Goodwin FK. "Cade's disease" and beyond: misdiagnosis, antidepressant use, and a proposed definition for bipolar spectrum disorder Can J Psychiatry 2002; 47: 125-34.

11 Sharma V, Khan M, Smith A. A closer look at treatment resistant depression: is it due to a bipolar diathesis? J Affect Disord 2005; 84: 251-7.

12 Huang KL, Su TP, Chen TJ, Chou YH, Bai YM. Comorbidity of cardiovascular diseases with mood and anxiety disorder: a population based 4-year study. Psychiatry Clin Neurosci 2009; 63: 401-9.
13 National Health Research Institutes. National Health Insurance Research Database, Taiwan. NHRI, 2007 (http://w3.nhri.org.tw/nhird//en/ Research.html).

14 National Center for Health Statistics. International Classification of Diseases (9th Rev. Clinical Modification) (ICD-9-CM). NCHS, 2010 (http://www.cdc.gov/ nchs/icd/icd9cm.htm).

15 Correa R, Akiskal H, Gilmer W, Nierenberg AA, Trivedi M, Zisook S Is unrecognized bipolar disorder a frequent contributor to apparent treatment resistant depression? J Affect Disord 2010; 126: 10-8.

16 Hantouche EG, Akiskal HS, Lancrenon S, Allilaire JF, Sechter D, Azorin JM, et al. Systematic clinical methodology for validating bipolar-II disorder: data in mid-stream from a French national multi-site study (EPIDEP). J Affect Disord 1998; 50: 163-73.

17 Goodwin G, Fleischhacker W, Arango C, Baumann P, Davidson M, de Hert M, et al. Advantages and disadvantages of combination treatment with antipsychotics: ECNP Consensus Meeting, March 2008, Nice. Eur Neuropsychopharmacol 2009; 19: 520-32.

18 Papakostas Gl. Managing partial response or nonresponse: switching, augmentation, and combination strategies for major depressive disorder. J Clin Psychiatry 2009; 70 (suppl 6): 16-25.

19 Papakostas GI. Pharmacologic and therapeutic strategies in treatmentresistant depression. Switching antidepressants vs. conventional augmentation strategies. CNS Spectr 2009; 14: 11-4.

20 Shelton RC, Osuntokun O, Heinloth AN, Corya SA. Therapeutic options for treatment-resistant depression. CNS Drugs 2010; 24: 131-61.

21 Dudek D, Rybakowski JK, Siwek M, Pawłowski T, Lojko D, Roczan R, et al. Risk factors of treatment resistance in major depression: Association with bipolarity. J Affect Disord 2010; 126: 268-71.

22 Akiskal HS, Maser JD, Zeller PJ, Endicott J, Coryell W, Keller M, et al. Switching from 'unipolar' to bipolar II. An 11-year prospective study of clinical and temperamental predictors in 559 patients. Arch Gen Psychiatry 1995; 52: 114-23.

23 Holma IAK, Holma KM, Melartin TK, Isometsä ET. Maintenance pharmacotherapy for recurrent major depressive disorder: 5-year follow-up study. Br J Psychiatry 2008; 193: 163-4.

24 Holma KM, Melartin TK, Holma IAK, Isometsä ET. Predictors for switch from unipolar major depressive disorder to bipolar disorder type I or II: a 5-year prospective study. J Clin Psychiatry 2008; 69: 1267-75.

25 Angst J, Sellaro R, Stassen HH, Gamma A. Diagnostic conversion from depression to bipolar disorders: results of a long-term prospective study of hospital admissions. J Affect Disord 2005; 84: 149-57. 